The Waxing and Waning of Mnemic Neglect

\author{
Bettina Zengel \\ University of Southampton \\ Brett M. Wells John J. Skowronski \\ Northern Illinois University
}

\begin{abstract}
Author Note
Brett M. Wells is now at Talent Plus Inc. (Lincoln, NE). This article contains studies from his dissertation, as well as additional data provided by the article's first author. The article's third author supervised both sets of studies. For their assistance we thank all the dissertation committee members, as well as the undergraduate students who helped to collect, code, and analyze the data reported in the present article.

Correspondence concerning this article should be addressed to Bettina Zengel, Centre for Research on Self and Identity, School of Psychology, Southampton SO17 1BJ, United Kingdom, Voice: +44 (0)23 80594584, FAX: +44 (0)23 80593328, E-mail: bettina.zengel@ gmail.com
\end{abstract}

() 2018, American Psychological Association. This paper is not the copy of record and may not exactly replicate the final, authoritative version of the article. Please do not copy or cite without authors permission. The final article will be available, upon publication, via its DOI: 10.1037/pspa0000124 


\begin{abstract}
The mechanisms underlying mnemic neglect $(\mathrm{MN})$ and the conditions under which it waxes and wanes are not yet fully understood. The research in this article examined conditions during both encoding and recall that could potentially moderate the $\mathrm{MN}$ effect and that could provide cues about the cognitive mechanisms that contribute to the effect. Results showed that MN: (a) emerged after recall was delayed (Study 1); (b) could not be attributed to differential behavior looking time (Study 2); and (c) did not emerge under cognitive load (Study 3); and (d) was not linked to the perceived extremity, importance or evaluations of the behaviors. However, how informative the behaviors were perceived for personality may contribute to the effect (Study 4). Finally, results from Study 3 and Study 4 showed that when participants were cognitively occupied during encoding, the MN effect waned. Implications of these collective findings for the MN phenomenon were discussed.
\end{abstract}

Keywords: mnemic neglect, loss of mnemic neglect, self-protection, self-enhancement, selfmemory 


\section{The Waxing and Waning of Mnemic Neglect}

One of the keys to happiness is a bad memory — Rita Mae Brown

Most people have a positive self-concept. Some cognitive processing mechanisms, such as preferential processing of information that is consistent with the positive self, will act to maintain this positive view of the self. However, it is also thought that an individual is motivated to maintain a positive self. Indeed, it is often proposed that people are motivated to both enhance the self and to defend the self, and that these motivations tend to be relatively powerful and pervasive (for an overview of self motivations, see Sedikides \& Strube, 1997). One way in which this motivation to enhance and protect the self may manifest is in selective memory. Self enhancement motivation and self-protection motivation may work to bias selfmemory to promote self-enhancing memories and to eliminate self-threatening memories (for example, see Skowronski, 2011).

The memory implications of motivated self-protection have been explored in a series of laboratory studies (see Sedikides \& Green, 2000) that have documented mnemic neglect (MN): The tendency for a person to selectively forget newly-encountered information that threatens their positive self-concept. In such studies people read a series of behaviors. The behaviors: (a) are said to describe either the experimental participant or a fictional person named Chris; (b) are either positive or negative, and (c) each either imply a trait that is relatively important to the participant's self-concept (e.g., "would borrow other people's belongings without their knowledge," which implies untrustworthiness) or imply a trait that is not especially important to the participants' self-concept (e.g., "would constantly talk about how much stuff there is to be done," which implies complaining). Often, the behaviors are portrayed as feedback (e.g., you 
took a personality test, and based on the results, these are behaviors that the test predicts you could do; Chris took a personality test, and based on the results, these are behaviors the test predicts that Chris could do). Later, in a free recall task, participants try to recall the behaviors. The recall data typically show that a participant exhibits relatively poor recall for negative behaviors that imply traits important to their self-concept, but only when these behaviors are depicted as those that could be performed by the participant, not when the behaviors are portrayed as those that could be performed by Chris. This selectivity in recall is mnemic neglect. Explaining the Mnemic Neglect Effect

In considering the phenomenon of MN, Sedikides and Green (2000) suggested that an individual is motivated to maintain a positive self-concept. As a result, individuals strategically neglect information that strongly threatens their self-concepts.

The word "neglect" implicates processing: One key assumption underlying the standard explanation for $\mathrm{MN}$ is that people process threatening behaviors shallowly (Brown \& Craik, 2000; Craik, 2002). For example, Sedikides, Green, and Pinter (2004) suggested that when the behaviors threaten central aspects of an individual's self-concept (as compared to behaviors that threaten peripheral aspects of an individual's self-concept), the individual will prematurely stop processing this information. Thus, because of this shallow processing, these self-threatening behaviors are at a disadvantage when it comes to recall.

A second assumption thought to underlie $\mathrm{MN}$ is that this shallow processing works in a specific manner: It does not allow the new information to be linked to other self-relevant information in memory, such as a person's memory of their past behaviors (see Pinter, Green, Sedikides, \& Gregg, 2011). The presence of such links are thought to be crucial to the ability to remember information in a free recall task. Ultimately, then, poor memory for newly 
encountered self-threatening information (mnemic neglect) is thought to occur because, as a result of shallow processing, in a search of memory that occurs during the free recall task there are relatively few routes that lead to the self-threatening behaviors.

\section{Empirical Evidence for the Motivated Processing Model}

The MN processing model has been supported by several sets of results. One set of studies compared two kinds of memory for self-threatening information (Green, Sedikides, \& Gregg, 2008): free recall performance versus recognition performance. Results from these studies show that MN occurs in free recall performance, but not in recognition memory performance. Recognition memory performance does not depend on links among items in memory, as does free recall performance. Hence, these results support the idea that MN occurs because self-threatening information is poorly integrated into the memory system, and not because the information was: (a) never stored to begin with (e.g., because of total inattention), or (b) that the information may have been stored in memory, but the memory trace for the event is gone.

The standard MN processing model has also been supported by findings contrasting memory results obtained in typical processing conditions with results obtained in restricted-time processing conditions (Sedikides \& Green, 2000, Experiment 3). When participants have limited time to process each behavior description, the $\mathrm{MN}$ effect in free recall dissipates. In theory, this loss of MN (a term introduced by Zengel, Skowronski, Valentiner, \& Sedikides, 2015) occurred because the limited processing time caused all behaviors to receive shallow processing, thus eliminating the selective processing mechanism thought to produce MN.

A third set of studies (Pinter, et al., 2011) focused on how MN is related to the extent to which self-threatening information is linked to an individual's existing self-concept. Separation- 
driven thought is theorized to spontaneously occur when new information threatens an individual's self-concept. Pinter et.al. directly tested this idea. When participants were explicitly instructed to separate self-threatening information from the self, the usual MN effect emerged. However, loss of MN emerged when participants were directly instructed to integrate self-threatening information into their self-concepts. Data also showed that there was an impairment in free recall for self-enhancing behaviors when people were specifically asked to separate those behaviors from the self-concept. These multiple results appeared only in a free recall task, not in a recognition task. Collectively, then, the Pinter et al. results supported the idea that free recall for newly encountered information is affected by whether people think about the information in a way that integrates it into, or separates it from, the self-concept. Poor free recall occurs when information is not well-integrated into the memory system, as is thought to spontaneously occur with self-threatening information.

\section{The Goals of Our Studies}

In the present article we report results from a number of new MN studies. These studies simultaneously pursued four goals. The first follows from the results of studies that show that the emergence of $\mathrm{MN}$ is sensitive to conditions (broadly construed, including memory tasks, instructions, processing opportunities, individual differences, and stimuli). For example, a loss of MN is observed when: (a) behaviors are seen as reflecting malleable traits instead of fixed traits (Green, Pinter, \& Sedikides, 2005), (b) negative behaviors are not seen as diagnostic of self-relevant negative traits (Green \& Sedikides, 2004), and (c) a self-improvement motive is subtly activated (Green, Sedikides, Pinter, \& Van Tongeren, 2009). MN is also lost for participants exhibiting anxiety, dysphoria, and schizotypy, and who exhibit a repressive coping style (Saunders, 2011, 2012; Saunders, Vallath, \& Reed, 2015; Saunders, Worth, \& Fernandes, 
2012; Zengel et al., 2015). Moreover, $\mathrm{MN}$ is absent in tests of recognition memory, and the emergence of $\mathrm{MN}$ in free recall is sensitive to the manner in which the behaviors are recorded in the free recall task (Newman, Sapolsky, Tang, \& Bakina, 2014). Following from these MN moderation studies, one goal of the present studies is to expand on the corpus of knowledge that details when MN occurs, and when it does not.

Some of the conditions that moderate the emergence of mnemic neglect are relevant to our second goal: To better understand some of the cognitive mechanisms that contribute to $\mathrm{MN}$. As in the Sedikides and Green (2000) and Green et al. (2008) free recall vs. recognition studies, mechanisms can sometimes be implied by the circumstances under which $\mathrm{MN}$ is observed (free recall) and in which it is lost (recognition). In other cases, as in Pinter et al. (2011), mechanism information can come directly from studies in which participants are asked to process the stimulus behaviors in different ways (inclusion in the self vs. exclusion from the self), or by approaches such as measuring aspects of the ways in which the stimulus behaviors are processed (e.g., assessing reading time). However, despite the progress that has been made so far, there is much to learn about cognitive mechanisms that contribute to MN. One candidate mechanism locates the cause early in processing: As implicated by the perceptual defense idea (e.g., Erdelyi, 1974), people may simply not attend to information that threatens the self. Hence, the memory trace for that information may be weak or absent, which can produce poor recall. A second candidate is reflected in the "standard" explanation for mnemic neglect, which suggests that its emergence reflects a causal chain: Lack of elaboration during behavior encoding causes few links to be formed to the memory of a self-threatening behavior, which makes such behaviors hard to find during the memory search that occurs during a free recall task. The studies that we report in this article attempt to provide evidence that can help to resolve this theoretical debate. 
The third goal of the studies described in this article is to explore the scientific credibility of the MN effect. Elements of science, including psychology, have recently been criticized for the supposed inability to replicate some of its phenomena (e.g., Schooler, 2014). Indeed, the scientific status of a phenomenon is enhanced when it is replicated: (a) often, (b) by different research teams, and (c) using multiple methods. Our perception is that the phenomenon of MN could use bolstering in these areas. There are relatively few empirical papers that explore the phenomenon of MN (a Psychinfo search conducted on Dec. 6, 2017 yielded only 22 publications in response to a search using the term "Mnemic Neglect"), and many of those papers have been produced by the Sedikides and Green research team (for a review, see Sedikides, Green, Saunders, Skowronski, \& Zengel, 2016). Only a handful have been produced by other scholars (for recent and representative publications, see Jones \& Brunell, 2014; Newman, Eccleston, \& Oikawa, 2017; Newman, Nibert, \& Winer, 2009; Saunders, et al., 2015). Thus, in the present article we sought to explore the scientific status of the $\mathrm{MN}$ construct by assessing its replicability, by documenting the conditions that influence its replicability, and by doing so in a context that is independent of the Sedikides and Green laboratories. ${ }^{1}$

The fourth goal underlying the studies that we report in the present manuscript is to shed light on the general issue of how the self influences memory, both a major and long-standing interest across many areas of psychology (e.g., see Klein, 2012). For example, some have wondered whether bias can simply be induced by an item's valence, asking whether people generally have better memory for the good in their lives or for the bad in their lives (see Skowronski, 2011). Others have wondered whether there is a self-consistency bias, asking whether people better remember those things that fit their self-conceptions or those that diverge from their self-conceptions (e.g., Thompson, Skowronski, Larsen, \& Betz, 1996). The studies 
that we describe in this article can speak to these issues. However, the MN paradigm provides a relatively unique approach to research in these areas. Because much of the existing research in these areas uses real-world memories, it is characterized by potential confounds between the characteristics of stimuli and the content of stimuli. These confounds may account for why research results exploring these questions often yield inconsistent results. The advantage of using the MN paradigm to address these questions is that it can bypass these potential confounds: Critical comparisons in the MN paradigm involve memory for the exact same behaviors as they describe the self or as they describe the fictional Chris.

Moreover, enhanced understanding of the causes of these kinds of memory biases that might be provided by MN studies has considerable practical importance. That is, it has often been suggested that people can only function optimally when they maintain a positive life outlook, and the maintenance of such an outlook is thought to be helped by the minimization of negative memories (e.g., Skowronski, 2011). Indeed, health psychology and clinical psychology are rife with examples of individuals whose functioning is impaired by negative memories, as in the case of PTSD (e.g., Schnurr \& Jankowski, 1999). Hence, MN research in general, which include the studies described in the present article, can provide critical experimental evidence about the causes of those self-related memory biases that can affect the extent to which an individual can maintain functionality in the real world.

\section{Study 1}

An initial study used a version of the MN paradigm developed by Sedikides and Green (2000). In this version of the paradigm participants read behaviors and are asked to consider them to be real. Some participants are asked to consider themselves as the enactor of the behaviors, but others are told that a fictional person named Chris is the enactor. Later, behavior 
memory is assessed via both a free recall task and a recognition task (see Green, et al., 2008). Pretesting conducted by Sedikides and Green (2000) showed that some of the negative behaviors that participants read (untrustworthy behaviors, unkind behaviors) are especially threatening to participant self-conceptions. If past free recall results are reproduced by our study, these should evince $\mathrm{MN}$, which should not emerge in the recognition data.

However, MN studies generally assess memory after only a short delay. In one set of conditions, we followed this procedure, asking participants to engage in a free recall task after only a 2.5 min filled delay. However, a second group of participants waited a full $48 \mathrm{hr}$ before returning to the study to complete the memory tasks. This manipulation obviously was intended to assess the temporal durability of the $\mathrm{MN}$ effect. The $48 \mathrm{hr}$ delay was selected because forgetting curve data (e.g., Loftus, 1985) suggests both that recall rates should decrease relative to the 2.5 min condition, but that there is some chance that a reasonable level of post-delay recall would remain. Hence, in our view a plausible outcome after a $48 \mathrm{hr}$ delay was that memory for all behaviors would decline, but that the MN effect would remain: Recall rates for important negative behaviors would still be higher when the behaviors described Chris then when the behaviors described the self.

However, it is also the case that MN might dissipate entirely after a $48 \mathrm{hr}$ delay. This might occur if memory for the all behaviors dissipates rapidly with the passage of time, thus eliminating the MN effect. This rapid memory loss might occur if the mnemic neglect paradigm produces very weak memories: Weak memories are quickly forgotten (Loftus, 1985). More intriguing than this general memory loss idea is the possibility that immediate memory for important negative self-descriptive behaviors is temporarily suppressed by the arousal prompted by encountering such behaviors (see Roozendaal, 2002). The passage of time ought to eliminate 
this arousal-prompted forgetting, so that with the passage of time recall for important negative self-descriptive behaviors might rebound to match the levels exhibited by those same important negative behaviors that describe Chris. The emergence of this latter effect would thus suggest that current thinking about the processes that produce mnemic neglect is not correct.

\section{Participants and Design}

Workers from Amazon's Mechanical Turk ${ }^{2}$ (MTurk) were recruited $(N=150)$. This sample size was determined via a power analysis ${ }^{3}$. Each was compensated with US \$2.00. Recruited workers were restricted to those who lived in the United States and who demonstrated high worker quality (at least a 95\% approval rating on previously submitted work). This targeted recruitment was used for all studies in this manuscript using MTurk. On agreeing to participate, workers were routed to Survey Monkey (http://www.surveymonkey.com), which presented all stimuli and recorded all responses. Using a splash page, participants were routed via a random assignment scheme to web pages that began the protocols for each of the four betweenparticipants conditions (Chris / delay; Chris / no delay; self / delay; self / no delay). Data from four participants were omitted from all analyses because they simply skipped through the experiment and failed to answer any questions.

\section{Procedure and Materials}

The procedure was adapted from prior research (e.g., Green, et al., 2005) and consisted of an encoding phase, a distracter task, the free recall task, and the recognition task. The task order was constant across all participants.

Encoding phase. Participants viewed at their own pace 32 behavioral statements. The back-button was disabled to prevent participants from returning to previous behaviors once they had read them and moved on to the next behavior statement. These behavior statements were 
identical to those used by Sedikides and Green (2000). Half of the behaviors that participants encountered were negative, and half were positive. Furthermore, half of the behaviors pertained to trait dimensions that are generally central to peoples' self-conceptions (trustworthiness, kindness), and the other half of the behaviors pertained to trait dimensions that are generally peripheral to peoples' self-conceptions (modesty, uncomplaining). Studies examining MN have used these behaviors and trait dimensions extensively (e.g., Green, et al., 2005; Green \& Sedikides, 2004; Green, et al., 2008; Newman, et al., 2009).

Participants (a) were told that the behaviors were to be thought of as coming from someone who knew the referent (self or Chris) well, and (b) were asked to consider the behaviors as real. For each participant, these behaviors were presented in a unique random order that was determined by the web pages. For half of the participants, these behaviors were said to directly pertain to each participant (i.e. the referent was the self). For the other half of participants, these behaviors were said to pertain to someone (not the participant) named Chris (i.e. the referent was Chris).

Distracter task. Participants were given two-and-a-half minutes to list as many of the United States as they could think of.

Delay condition procedures and instructions. Participants in a no delay condition completed, immediately following the distracter task, a behavior recall task followed by a behavior recognition task.

In the delayed recall condition, after immediately completing the distracter task, participants were informed that in 48 hours they would complete the second part of the experiment. They were given a Survey Monkey website link that became activated 48 hours after finishing the distracter task. To minimize attrition and to prompt task completion, 
participants in this condition received a reminder via e-mail. Despite the reminder, 34 people in the 48-hr delay condition who completed the first study phase did not attempt the second study phase, so they provided no data for the experiment. Moreover, eight participants in the 48-hr delay condition failed to complete either the behavior recall task or the behavior recognition task, so whatever data they did provide were excluded from analyses. Participants in the 48-hr delay condition who finished the protocol completed the same free recall task and behavior recognition task that were completed by participants in the no delay condition.

Behavior recall task. Participants were asked to list as many behaviors as they could remember, without worrying about verbatim reporting, for five minutes. They were provided with 32 lines on one computer screen to provide their reports.

Behavior recognition task. When participants finished the behavior recall task, they engaged in a behavior recognition task. The behavior recognition task had two purposes. The first is concerned with specifying conditions under which MN may occur and conditions in which it may be lost. Past research results show that $\mathrm{MN}$ is lost on an immediate recognition task, but we thought that it was possible that MN might appear on the delayed recognition task. This possibility was considered because in prior MN studies, immediate recognition memory for all behaviors was quite good. We speculated that some degree of impairment of recognition memory might be necessary for $\mathrm{MN}$ to emerge in the recognition memory task. Because recognition memory should degrade with delay, the inclusion of a delayed recognition task assessed this possibility. Second, to our knowledge, only two sets of experiments have examined whether MN emerges in recognition tasks (Green et al., 2008; Pinter et al., 2011). Hence, in our view, replication is warranted. 
In the recognition task participants were shown 64 behaviors. The ordering of behaviors was randomly determined for each participant by the web pages. Half of these behaviors were identical to the behaviors presented during the encoding phase; the rest were lures that implied the same traits as those implied by the actual encoding task behaviors, but that were never read during the encoding phase. These lures were taken from Green et al. (2008), and were designed to closely parallel the original behaviors. During the recognition test, participants were asked to indicate whether behaviors were "old" (i.e., behaviors were previously read during the encoding phase) or "new" (i.e., behaviors were never read during the encoding phase).

\section{Results}

The experiment was conducted as a 2 (Recall Delay: 2.5 min vs. $48 \mathrm{hr}) \times 2$ (Behavior Referent: self vs. Chris) $\times 2$ (Trait Type: central vs. peripheral) $\times 2$ (Behavior Valence: positive vs. negative) mixed-model factorial design. The first two variables were between-subjects and the last two were within-subjects. This design was used in separate analyses of the free recall data and of the recognition data. To save space, our discussion of results focuses on those effects yielded by the analyses that are crucial to the manuscript's goals. A full presentation of results from all analyses is available from this article's first author.

Free recall data. Free recall items recorded for each participant who finished the experimental protocol were screened. In many mnemic neglect studies two raters have been used to determine if the gist of the recalled behavior matches the originally presented behavior. However, agreement rates have been high (typically over 90\%) and most of the disagreements were regarding intrusions or corrections that involved accidentally assigning the wrong code to a behavior. Our studies have therefore relied only on one coder who was careful to avoid these problems. 
Following the procedure of Sedikides and Green (2000), intrusions were removed from the data prior to analyses. Intrusions included the following: (a) recalling a behavior that was not presented, (b) writing the same behavior twice, and (c) changing the valence of a recalled behavior. Intrusions represented approximately thirteen percent of the recalled items. This percentage exceeds that usually observed in MN studies (roughly five percent; J. Green, personal communication, January 28,2011$)$. One might guess that the intrusion rate was a consequence of the internet-based sample and method used in Study 1. Sedikides and Green (2000) dropped from analyses data from participants whose recall protocol exhibited three or more intrusions. We did the same: Eight participants were excluded from analyses for this reason. Additionally, data from one participant in the no delay condition was excluded from analyses because that participant reported that he/she could not recall a single behavior (though analyses yielded similar results regardless of whether or not this participant was retained). These same exclusion criteria are used throughout the studies reported in this paper.

Following all participant deletions, the number of participants in each betweenparticipants cell of the study was as follows: Chris/delay $=17$, Chris/no delay $=34$, self $/$ delay $=$ 12 , self/no delay $=32$. Due to attrition and the greater tendency to not recall any behaviors after a 48-hr delay, the sample size was relatively small. However, the goal of 60 participants derived from our power analysis was met. We also considered the possibility that participants had a legitimate reason not to recall any behaviors after the 48-hr delay (weak original memories). Thus, we conducted an alternative analysis that included the 34 participants who recalled no behaviors in the delay condition. Inclusion of these participants did not alter the MN-relevant pattern of results. Note that in the section below we report the data with the no-recall participants excluded. This was done to maintain consistency in the exclusion rules used in 
Experiment 1 and in the other studies reported in this manuscript (which did not use the delay manipulation).

Following the procedures outlined by Sedikides and Green (2000), free recall data were scored using a general meaning (gist) criterion. The proportion of trials on which a behavior was scored as correctly recalled using this criterion was separately calculated for each participant for each cell of the Trait Type (central vs. peripheral) $\times$ Behavior Valence (positive vs. negative) within-subject matrix. For analysis, these proportions were entered into a Recall Delay $x$ Behavior Referent $\times$ Trait Type $\times$ Behavior Valence mixed model ANOVA.

The MN effect emerged. Sedikides and Green (2000) noted that one indication of MN lies in the Behavior Referent $\times$ Trait Type $\times$ Behavior Valence interaction. Our ANOVA yielded this exact interaction, $F(1,91)=4.59, p=.035, \eta_{p}{ }^{2}=.048$. The means for this interaction (see Table 1) evince MN: Compared to the recall rate for central/negative behaviors that described Chris, recall rates for central/negative behaviors that described the self were low $[t(93)=-2.58$, $\left.p=.012, \eta_{p}{ }^{2}=.067\right]$. Moreover, this effect was evident both at immediate recall and at delayed recall (non-significant Delay $\times$ Behavior Referent $\times$ Trait Type $\times$ Behavior Valence interaction, $\left.F(1,91)=0.32, p=.576, \eta_{p}{ }^{2}=.003\right)$. Thus, $\mathrm{MN}$ is not an ephemeral phenomenon, but is evident in long-term memory.

The fact that delay did not moderate the Behavior Referent $\times$ Trait Type $\times$ Behavior Valence interaction was not a consequence of an ineffective delay manipulation. The ANOVA yielded a significant Delay $\times$ Trait Type interaction, $F(1,91)=8.68, p=.004, \eta_{p}{ }^{2}=.087$, as well as a main effect for Delay, $F(1,91)=18.92, p<.001, \eta_{p}{ }^{2}=.172 . \quad$ As is evident from Table 1 , the means for the interaction show that for peripheral behaviors, more behaviors were recalled in the no delay condition $(M=.16, S D=.15)$ than in the delay condition $(M=.07, S D=.10)$, 
$F(1,93)=7.60, p=.007, \eta_{p}{ }^{2}=.076$. This pattern was especially pronounced for central behaviors (no delay $M=.35 S D=.18$; delay $M=.17, S D=.11$ ), $F(1,93)=23.00, p<.001$, $\eta_{p}^{2}=.198$

Behavior recognition data. Following the procedures outlined by Green et al. (2008), recognition data were analyzed using signal detection theory (Banaji \& Greenwald, 1995; Stanislaw \& Todorov, 1999; Swets, 1996). For old-new recognition tests, four types of answers are possible: An old behavior can be correctly identified as old (a hit); a new behavior can be correctly identified as new (a correct rejection); an old item can be mistakenly classified as new (a miss); and a new item can be mistakenly classified as old (a false alarm). Conceptually, recognition accuracy $(\delta)$ refers to the extent to which one can both correctly identify old behaviors as old and correctly identify new behaviors as new.

In Study 1 accuracy rates were derived by converting mean hits and mean correct rejections into proportions, and then averaging the result. These $\delta$ values were separately calculated for each participant for each cell of the Trait Type (central vs. peripheral) $\times$ Behavior Valence (positive vs. negative) within-subject matrix. The values were entered into a mixedmodel ANOVA that included these variables, as well as the between-subjects variables of behavior referent (self vs. Chris) and recall delay ( $2.5 \mathrm{~min}, 48 \mathrm{hr}$ ).

No MN in behavior recognition. Of main importance in the analysis is whether evidence of MN emerged in the recognition data. It did not (see Table 2). The Behavior Referent $\times$ Trait Type $\times$ Behavior Valence interaction that is thought to be one indicator of MN was not significant, $F(1,91)=.00, p=.995 \eta_{p}^{2}<.001$. We also considered the possibility that MN in recognition might occur only after a delay. As reflected in the nonsignificant Delay $\times$ Behavior 
Referent $\times$ Trait Type $\times$ Behavior Valence interaction, $F(1,91)=.11, p=.747, \eta_{p}{ }^{2}=.001$, the data did not support this possibility.

The absence of $\mathrm{MN}$ in the recognition data cannot be explained by the idea that the manipulations were ineffectual. For example, replicating previous research, central behaviors were better recognized than peripheral behaviors, $F(1,91)=9.66, p=.003, \eta_{p}{ }^{2}=.096$.

Moreover, there was a main effect for delay, $F(1,91)=8.92, p=.004, \eta_{p}{ }^{2}=.089$, and a marginal Delay $\times$ Behavior Referent $\times$ Behavior Valence interaction, $F(1,91)=3.47, p=.066, \eta_{p}^{2}=.037$. The means for this interaction do not inform MN, simply suggesting that the effect of delay on recognition accuracy was especially large for negative behaviors that described Chris.

\section{Discussion}

Study 1 replicated prior MN results, showing that it occurs in a free recall task, but not in a behavior recognition task. It extended these prior results by showing: (a) that $\mathrm{MN}$ emerges in free recall after a $48 \mathrm{hr}$ delay between exposure to the behaviors and engagement in the free recall memory task; (b) that the loss of $\mathrm{MN}$ in recognition occurs after a $48 \mathrm{hr}$ delay between exposure to the behaviors and engagement in the recognition task, and (c) the effects described in (a) and (b) emerge from a sample of Survey Monkey workers who completed the tasks in a nonlaboratory setting (most previous studies explored university students in laboratory settings).

However, one possible limitation of our findings was that the recognition task was administered after the recall task. It should be noted that this procedure was used because it duplicated the procedure of Green et al. (2008), and our main focus was on the recall measure. We therefore did not alter the sequence of when the recognition assessment occurs. However, the use of this fixed task ordering does raise the possibility that performance on the recognition task was affected by prior performance of the free recall task. Hence, it is possible that if the 
recognition task were to be administered by itself, a $\mathrm{MN}$ effect might emerge with the recognition assessment. Because of the theoretical significance of such a result, future studies ought to pursue this possibility.

\section{Study 2}

The results from Study 1 suggest that MN is not an ephemeral phenomenon, but is produced by processes affecting long-term memory. Study 2 pursued evidence regarding some of the plausible processes that might produce such long-lasting effects.

The standard explanation for MN implicates depth of self-related processing: Threatening information is not processed deeply and is not linked to self-knowledge, so it is not well-remembered in a free recall task. However, the exact locus of this shallow processing requires additional specificity. One possibility is that this shallow processing could reflect a relative lack of attention to threatening stimuli might be differential attention. As in the idea of “perceptual defense” (e.g., Erdelyi, 1974; also see Ochsner \& Gross, 2005; Pool, Brosch, Delphanique, \& Sander, 2016), when a stimulus is detected as threatening, people may quit attending to the stimulus or divert attention to another stimulus. This implies that people should spend relatively little time looking at self-threatening information. This fits with an early view articulated by Sedikides and Green (2000): “central negative information is recalled poorly because, at least in part, such information receives the allocation of minimal processing time" (p. 914). If this minimal allocation occurred when people initially encountered a behavior, then one would expect patterns in behavior reading time to largely mirror patterns in behavior recall.

A second possible alternative contributor to $\mathrm{MN}$ lies in differential encoding ease.

Because self-important negative information is inconsistent with a person's usually-positive selfview, such information may be especially difficult to encode. This idea comes from the person 
memory literature, which suggests that reading time is especially long for expectancyinconsistent behaviors and that this reading time effect is a plausible mediator of recall (Bargh \& Thein, 1985; Belmore, 1987; Stern, Marrs, Millar, \& Cole, 1984). This implies that people should spend an especially long time processing self-threatening behaviors. However, it is also plausible that this processing time effect might extend to any negative stimuli, not just to selfimportant negative stimuli (e.g., Fiske, 1980). Negative stimuli are generally thought to be atypical and unexpected, so such stimuli might be expected to require additional processing above and beyond its degree of self-importance. Hence, if there is a slowdown in processing of negative information, it is plausible that it might be observed for all negative behaviors, not just for negative behaviors that have implications for central traits.

Study 2 explored these alternative ideas by using a standard MN paradigm and measuring the length of time that participants spent reading the behaviors.

\section{Participants and Design}

Students $(N=114)$ enrolled in an introductory psychology course at Northern Illinois University participated. Compensation was partial credit toward completion of a course research option.

The experiment was conducted as a 2 (Behavior Referent: self vs. Chris) $\times 2$ (Trait Type: central vs. peripheral $) \times 2$ (Behavior Valence: positive vs. negative) mixed factorial design. The first variable in the design was between-subjects and the last two variables were within-subjects. The behavior presentation orders were randomly determined for each participant by the DirectRT research software (Empirisoft, 2004) used to conduct the experiment.

\section{Procedure and Materials}


The procedure and materials duplicated Study 1, except: (a) only behavior recall, and not behavior recognition, was included after the distracter task; (b) the recall delay manipulation was not used; (c) the study was conducted in the laboratory using DirectRT software; (d) the time spent with each behavior before moving on to the next behavior was measured and recorded by the software; and (e) in the recall task participants received a response box to enter the behaviors they could recall one at a time, with the response box being cleared after each behavior was submitted. This last change matches recommendations from Newman and colleagues (2014) to instruct participants to recall only one behavior at a time without the possibility of going back and reading previous entries. According to the Newman et al. (2014) findings, this instruction, when accompanied by a five min recall period, produced typical MN effect. Newman et al. speculated that such procedures bypass output interference that might prevent the emergence of MN.

\section{Results}

Recall data. Following the data cleaning procedure of Study 1 and Sedikides and Green (2000), participants who recalled no valid behaviors or who exhibited more than three intrusions were removed from the data set $(n=25)$. Thus, the final sample consisted of 89 participants. The exclusion of participants did not vary by referent.

The free recall responses from these participants were coded using a gist criterion following the procedures outlined by Sedikides and Green (2000) (the same procedures used in Study 1). The proportion of correctly recalled behaviors was separately calculated for each participant for each cell of the Trait Type (central behaviors vs. peripheral behaviors) $\times$ Behavior Valence (positive behaviors vs. negative behaviors) within-subject matrix. These proportions were entered into a mixed-model ANOVA that reflected the experiment's factorial design. 
Replication of the MN effect. Sedikides and Green (2000) noted that one possible indicator of MN lies in the Behavior Referent $\times$ Trait Type $\times$ Behavior Valence interaction. Our results evinced this interaction, $F(1,87)=23.37, p<.001, \eta_{p}{ }^{2}=.212$ (see Table 3 ). Decompositions of the interaction confirm the presence of MN. The Referent $\times$ Behavior Valence interaction did not emerge for peripheral trait-relevant behaviors, $F(1,87)=1.46, p=$ $.230, \eta_{p}{ }^{2}=.017$. In comparison, the central trait-relevant behaviors evinced a significant Behavior Referent $\times$ Behavior Valence interaction, $F(1,87)=31.49, p<.001, \eta_{p}{ }^{2}=.266$. Furthermore, results of additional decompositions showed that for these central trait-relevant behaviors there was not a significant difference in referent-related recall for positive behaviors $(p$ $=.659)$. In comparison, the recall rate of self-referent negative trait-relevant central behaviors was significantly lower than the recall rate for Chris-referent negative trait-relevant central behaviors, $F(1,87)=28.21, p<.001, \eta_{p}^{2}=.245$.

The overall ANOVA yielded other significant effects. These included Trait Type $\times$ Behavior Valence $\left[F(1,87)=16.13, p<.001, \eta_{p}{ }^{2}=.156\right]$ and Behavior Referent $\times$ Behavior Valence $\left[F(1,87)=8.33, p=.005, \eta_{p}{ }^{2}=.087\right]$ interaction effects, and Behavior Valence $\left[F(1,87)=15.13, p<.001, \eta_{p}{ }^{2}=.148\right]$, Trait Type $\left[F(1,87)=120.71, p<.001, \eta_{p}{ }^{2}=.581\right]$, and Behavior Referent $\left[F(1,87)=14.68, p<.001, \eta_{p}{ }^{2}=.144\right]$ main effects. However, interpretations of all of these are qualified by the significant three-way interaction, so we forego discussion of these effects.

Behavior reading times. The mean time ${ }^{4}$ spent on each behavior during the behavior encoding phase was separately calculated for each participant for each cell of the Trait Type (central behaviors vs. peripheral behaviors) $\times$ Behavior Valence (positive vs. negative) within- 
subject matrix. These times were entered into a mixed-model ANOVA that reflected the experiment's factorial design.

Unlike the analysis of behavior recall, analysis of behavior reading times did not evince a Behavior Referent $\times$ Trait Type $\times$ Behavior Valence interaction $(p=.607$; see Table 4$)$. To be cautious, we also considered that reading time might have an effect regardless of the trait type: The distinction between central and peripheral is not always completely clear-cut (Zengel et al., 2015), and there is reason to believe that reading time effects might emerge for event valence, regardless of self-importance (e.g., Fiske, 1980). However, the Behavior Referent $\times$ Behavior Valence interaction was also not significant $(p=.681)$.

Instead, the analysis results evinced a significant Trait Type $\times$ Behavior Valence interaction, $F(1,87)=6.90, p=.010, \eta_{p}{ }^{2}=.073$. This interaction was decomposed by looking at behavior valence effects within each level of the trait type variable. For peripheral behaviors, reading time did not vary between peripheral positive behaviors and peripheral negative behaviors $(p=.538)$. However, in the case of central behaviors, reading time was longer for positive behaviors than for negative behaviors, $F(1,88)=18.82, p<.001, \eta_{p}{ }^{2}=.176$. The ANOVA also evinced a significant behavior valence effect $[F(1,87)=14.27, p<.001$, $\left.\eta_{p}{ }^{2}=.141\right]$ that was qualified by the Trait Type $\times$ Behavior Valence interaction effect. No other effects were significant.

\section{Discussion}

As in Study 1, in Study 2, the MN effect in free recall was replicated. The recall rate of self-referent negative trait-relevant central behaviors was significantly lower than the recall rate for Chris-referent negative trait-relevant central behaviors. 
Study 2 also assessed whether this effect could somehow be linked to the time people spent looking at the behaviors. People exhibited short looking times to central trait-relevant negative information, but did so equally whether the information described the self or described Chris. Despite this equivalence, the central-trait relevant information that described Chris was better recalled than the central trait-relevant information that described the self. Such results make it hard to argue that MN occurs because people avoid looking at self-threatening information.

The looking time data also make it hard to argue that MN occurs because the central traitrelevant behaviors are especially hard to understand when they implicate the self. People exhibited short looking times to central trait-relevant negative information, but did so equally whether the information described the self or described Chris. This result suggests that negative behaviors may have been especially easy to understand, but that this ease of understanding was not affected by whether the central trait-relevant behavior was descriptive of the self or Chris.

However, reading time is not a pure measure, and the sloppiness in the measure may hide multiple effects. Reading time is composed of the time it takes to physically read the behavior plus the amount of time it takes to draw upon existing knowledge to understand the behavior. These may work in opposing ways. A couple of examples may illustrate these opposing effects.

For example, when reading a self/central/positive behavior, a person could spend a relatively short amount of time (e.g., 2 s) reading the actual behavior because it is vivid and consistent with one's positive self-image (i.e., the behavior is processed with high fluency). Because of this, a relatively long amount of time (e.g., 2 s) could be spent on processing the behavior, linking it to both semantic knowledge and episodic memories. However, when reading a self/central/negative behavior, a person could spend a relatively long amount of time (e.g., $3 \mathrm{~s}$ ) 
reading the actual behavior because it is inconsistent with one's positive self-image (i.e., the behavior exhibits low fluency). Because of self-protection concerns, a relatively short amount of time (e.g., $1 \mathrm{~s}$ ) could be spent on processing the behavior and on linking it to semantic knowledge and episodic knowledge. Hence, reading time as measured in Study 2 could be identical for both self-affirming behaviors and self-threatening behaviors (e.g., 4 s), but the actual amount of processing time allocated to drawing on self-knowledge could vary across the behaviors (e.g., $2 \mathrm{~s}$ versus $1 \mathrm{~s})$.

Despite these qualifications, the data from Study 2 are clear: In the standard MN paradigm, MN is not directly linked to reading time. This result suggests (though does not conclusively demonstrate) that $\mathrm{MN}$ is not the consequence of differential attention to behaviors, nor is it a consequence of the difficulty of encoding behaviors.

\section{Study 3}

The explanation left standing after Study 2 is the "standard" MN explanation suggesting that $\mathrm{MN}$ is caused by the shallow processing accorded to important negative self-relevant events. One implication of this idea is that if one causes a participant to shallowly process all the behaviors received in the $\mathrm{MN}$ paradigm, loss of $\mathrm{MN}$ will emerge.

This idea was pursued in Study 3 via a cognitive load manipulation. Some participants completed the MN paradigm in the standard conditions. Others were given a secondary task to complete while trying to process the behaviors. The cognitive load induced by the secondary task should work to produce shallow processing of all behaviors. Accordingly, a loss of MN should be observed in the cognitive load condition. 


\section{Participants and Design}

Students $(N=94)$ enrolled in an introductory psychology course at Northern Illinois University participated. Compensation was partial credit toward completion of a course research option.

The experiment was conducted as a 2 (Cognitive Load: no cognitive load vs. cognitive load) $\times 2$ (Referent: self vs. Chris) $\times 2$ (Trait Type: central behaviors vs. peripheral behaviors $) \times$ 2 (Behavior Valence: positive behaviors vs. negative behaviors) mixed-model factorial design. The first two factors in the design were between-subjects; the latter two were within-subjects.

\section{Procedure and Materials}

With one exception, the materials and procedures used in this study duplicated those of Study 2. The exception was the inclusion of a cognitive load manipulation. One group of participants (randomly assigned) completed exactly the same task completed by participants in Study 2. The second group of participants (also randomly assigned) was given a secondary task to complete while they tried to process the behaviors: They tried to remember a six-digit number. For those in this condition, participants were first shown a computer screen displaying a six-digit number. A different number was used for each behavior encountered. On each trial participants were given five seconds to commit this number to memory. On a second screen, they were shown (for $8 \mathrm{~s}$ ) a single behavioral statement. The timing matched the ample processing condition in Sedikides and Green (2000, Experiment 3). On a third screen, participants were asked to reproduce the number from the first screen. Participants were instructed to remember the number to the best of their ability, and to reproduce it exactly as it appeared on the first screen (see Figure 1). This cognitive load manipulation has been shown to 
effectively minimize cognitive capacity and to interfere with stimulus processing (e.g., Wells, Skowronski, Crawford, Scherer, \& Carlston, 2011).

\section{Results}

Data recorded for each participant were subjected to a preliminary screening. Results of this screening led to the deletion of several participants from the sample. One participant was excluded from analyses because of a computer failure, seven participants were excluded from analyses because they failed to recall any behavior, and four participants were excluded from analyses because they recalled trait words as opposed to behaviors. Lastly, intrusions were removed from these data prior to analyses. Again following the procedures outlined by Sedikides and Green (2000), 15 participants were excluded from analyses because they recalled three or more intrusions. Intrusions represented approximately nineteen percent of the recalled items. Overall, data cleaning procedures led to the deletion of 26 participants. Thus, the final sample consisted of 67 participants. The exclusion of participants did not vary substantially between the referent groups or cognitive load groups.

The recall data were again coded as correct based on a gist criterion. Correct recall proportions were then calculated separately for each cell of the within-subjects design. These proportions were the dependent variables in a mixed-model ANOVA that reflected the experiment's factorial design.

Moderation of the MN Effect. Of main interest in the study was whether the presence of MN in free recall depended on cognitive load. It did (see Table 5 for behavior recall means and standard deviations).

The ANOVA yielded a number of significant effects (Cognitive Load $\times$ Behavior Referent $\times$ Behavior Valence $\left[F(1,63)=5.44, p=.023, \eta_{p}{ }^{2}=.080\right]$, Cognitive Load $\times$ Behavior 
Valence $\left[F(1,63)=5.44, p=.023, \eta_{p}{ }^{2}=.080\right]$, Cognitive Load $\times$ Trait Type $[F(1,63)=8.91$, $\left.p=.004, \eta_{p}{ }^{2}=.124\right]$, Trait Type $\left[F(1,63)=120.31, p<.001, \eta_{p}{ }^{2}=.656\right]$ and Cognitive Load $\left.\left[F(1,63)=32.34, p<.001, \eta_{p}{ }^{2}=.339\right]\right)$, but we focus on the effect of main theoretical importance: the significant Cognitive Load $\times$ Behavior Referent $\times$ Trait Type $\times$ Behavior Valence interaction, $F(1,63)=9.36, p=.003, \eta_{p}{ }^{2}=.129$. We thought that the loss of MN under cognitive load would be best illustrated by a series of decompositions that parsed this four-way interaction.

The first decomposition explored the data separately by the trait type variable. When peripheral traits were implied by behaviors, the Cognitive Load $\times$ Behavior Referent $\times$ Behavior Valence interaction was not significant $(p=.193)$. However, this same three-way interaction was significant when central traits were implied, $F(1,63)=10.49, p=.002, \eta_{p}{ }^{2}=.143$.

Decomposition by of this latter significant interaction by the behavior valence variable showed that for negative central behaviors the Cognitive Load $\times$ Behavior Referent interaction was significant, $F(1,63)=4.94, p=.030, \eta_{p}{ }^{2}=.073$. Further decomposition revealed that while self-referent central trait-implying negative behaviors trended towards being recalled less well than Chris-referent central negative behaviors in the no load condition, $F(1,34)=3.11, p=.087$, $\eta_{p}{ }^{2}=.084$, this was not the case under cognitive load $(p=.170)$. Thus, while the data in the no load condition replicated ${ }^{5}$ the $\mathrm{MN}$ effect, the effect was lost under cognitive load. In comparison, for positive central trait-implicative behaviors the Cognitive Load $\times$ Behavior Referent interaction approached significance, $F(1,63)=3.72, p=.058, \eta_{p}{ }^{2}=.056$. However, further decomposition indicated that both for the no load condition $(p=.244)$ and for the load condition $(p=.139)$ there was not a significant difference between recall of self-referent positive central behaviors and Chris-referent positive central behaviors. 


\section{Discussion}

When the conditions of Study 3 duplicated those in Study 2, MN emerged: central negative behaviors recalled especially poorly when those behaviors were self-referent but not Chris-referent. However, this MN effect was lost when participants tried to encode the behaviors under cognitive load. This was expected from the MN model: By eliminating the processing disadvantage of self-framed central trait-implying negative behaviors, $\mathrm{MN}$ should be lost. That is exactly what happened.

This result conceptually duplicates results from Sedikides \& Green (2000, Experiment 3). Their study produced loss of MN by restricting reading time. However, in both our Study 3 and in the Sedikides and Green study, it is possible that the loss of MN may not solely be due to behavior processing (formation of linkages to existing information in memory) as specified by MN theory. Poor memory caused by both minimal reading time and by cognitive load might simply come from the fact that the behaviors themselves may be incompletely read or understood.

This impaired reading/understanding issue can be bypassed by using manipulations that ensure that the behaviors are completely read and understood, but that still interfere with the kinds of processing that are thought to enable the behaviors to be linked to other information in long-term memory. Experiment 4 pursued this idea by asking participants to make ratings of the behaviors as they were encountered. To make their ratings participants must attend to, read, and understand the behaviors. However, despite attending to and processing the behaviors, the rating task may interfere with the processing that is necessary to link the behavior to long-term memory. A similar idea was described by Wyer and Carlston $(1979$, p. 133). They suggested that manipulations that caused perceivers to focus on the details of behavioral information 
interfered with the extent to which the behavior was integrated with other behavioral information in memory. Reasoning from the MN model, we thus expected that this interference should produce loss of MN by reducing the extent to which all behaviors (not just important negative self-relevant behaviors) could be linked to an individual's existing knowledge. This loss of MN should occur despite the fact that the ratings task ensured that individuals read and understood the behaviors.

\section{Study 4}

\section{Participants and Design}

Data for Study 4 were collected in three waves. In the initial wave of data collection, participants who were recruited for the study either read and evaluated (good/bad) each behavior (behavior evaluation condition), or read each behavior and rated several of the characteristics of each behavior (behavior rating condition). The behaviors that were read were the same as the ones used in Studies 1, 2, and 3. In response to reviewer feedback, a second wave of data collection occurred. In this second wave, additional subjects engaged in one of the same two tasks that were used in the initial wave. A third (MN replication) condition was used in this second wave of data collection. Participants assigned to this condition completed the standard MN paradigm. Hence, the data from the two waves of data collection looked for the emergence of $\mathrm{MN}$ in the $\mathrm{MN}$ replication condition, and the loss of $\mathrm{MN}$ in both the behavior evaluation condition and the behavior rating condition.

In the first wave of data collection a total of 200 participants were recruited through MTurk and paid $\$ 1.00$ (US) for their time. Eleven additional participants started the study but did not complete it and were not paid. Thus, any data they provided were discarded. 
Participants were asked to report some demographic information, such as their age. However, ethnicity was not assessed.

In first wave of data collection, ninety-seven participants were assigned to the behavior evaluation condition. However, three participants either provided ratings without variation throughout or they failed to complete the majority of rating items. Thus, any data they provided were discarded. The remaining 97 participants ranged in age between 18 and 65 years $(M=$ 35.62, $S D=12.57)$. Most participants were female $(62.9 \%)$.

Additionally, in the first wave of data collection, 96 participants were assigned to the behavior rating condition. Fifteen participants did not answer the demographic questions. The age of the demographic responders ranged from 18 to 65 years $(M=33.51, S D=11.92)$, and most $(55.2 \%)$ were female.

In the second wave of data collection, 450 participants were recruited through MTurk and paid $\$ .60$ (US) as compensation for their time. Because they either provided no ratings, failed to complete the majority of rating items, or said that they encountered technical difficulties as they tried to complete the study, 22 of these participants were excluded from the study. The remaining 428 participants ranged in age from 18 to 74 years $(M=38.54, S D=12.75)$. Most participants were female (64.9\%; with missing data of two participants) and Caucasian $(78.1 \%$; Black: 6.1\%, Asian: 4.9\%, Hispanic: 3.5\%, mixed ethnicities: $2.8 \%$; American without further ethnic distinction: $2.8 \%$; Native American: $0.9 \%$, other: $0.7 \%$ with data from four participants missing). Of these 428 participants, 148 were randomly assigned to the behavior evaluation condition, 133 were randomly assigned to the behavior rating condition, and 147 were randomly assigned to the $\mathrm{MN}$ replication condition. 
Thus, when data from both waves of data collection were combined, 245 were in the behavior evaluation condition, 233 were in the behavior rating condition, and 147 were in the MN replication condition.

\section{Procedure and Materials}

Participants in the $\mathrm{MN}$ replication condition completed a version of the mnemic neglect paradigm (see Study 1). However, to make the three conditions of Study 4 as equivalent as possible, in this version of the MN paradigm the behavior presentation was not automatically paced. Instead, after $6 \mathrm{~s}$ a "next" button became available and could be used to cause a new behavior to appear. This $6 \mathrm{~s}$ time seems to hit a "sweet spot" for self-paced processing. Feedback from participants who participated in some of the other MN studies that have been conducted in our lab indicated that times longer than $6 \mathrm{~s}$ were annoyingly long. More importantly, this $6 \mathrm{~s}$ minimum is comparable with values used in other studies, and ensures that participants in the MN condition would not speed through the display without reading the behaviors (see Sedikides \& Green, 2000, Study 3). In the Study 4 procedure, the behaviors were displayed with an "X" as placeholder for the referent (prior studies explicitly presented the behavior referent). Participants were instructed, while reading the behaviors, to replace $\mathrm{X}$ either with their own name (self-referent condition) or with the name "Chris" (Chris-referent condition) while reading the behaviors. In all conditions, after reading (or reading and rating) the behaviors, participants encountered the same distractor task and surprise recall task used in Study 1.

The behavior rating condition duplicated the methods and instructions used in the MN replication condition, but asked participants to provide ratings of the behaviors right after reading each. These ratings employed variations of the types of questions that were used to create and 
select the stimulus material for the mnemic neglect paradigm (Sedikides \& Green, 2000). Specifically, participants were asked to rate: (a) how negative/positive the behavior is $(-3=$ extremely negative, $+3=$ extremely positive), (b) how important to personality it is to perform/not perform the behavior $(-3=$ extremely important not to perform $;+3=$ extremely important to perform), and (c) how uninformative/informative the behavior is to personality (- 3 $=$ extremely uninformative, $+3=$ extremely informative). All ratings were performed with all scale points labeled using the scheme extremely very , slightly , neither nor slightly very , extremely

The behavior evaluation condition also duplicated the methods and instructions used in the $\mathrm{MN}$ replication condition, but asked participants to provide a rating of a behavior right after reading each. This was simply a rating of the extent to which a behavior was good or bad, and responses were made on the same kind of 7-point scale described in the prior paragraph.

\section{Results}

Data cleaning. The responses to the free recall task were again coded using a gist criterion. As with prior mnemic neglect studies, a total of 143 (37 from original data collection with behavior ratings; 22 from original data collection with evaluations; 84 from extended data collection) participants were excluded from the data analysis because they either did not recall any behaviors or their recall contained too many intrusions. After data exclusion, 191 participants remained in the behavior evaluation condition, 179 participants remained in the behavior rating condition, and 123 participants remained in the MN replication condition.

In response to a concern about a referent bias in these exclusions, we explored the extent to which these exclusions were balanced across the self-referent and Chris-referent conditions. Results of a chi-square test, $\chi^{2}(1)=4.58, p=.032$, indicated that more participants from the self 
condition (81 excluded vs. 231 not excluded) were excluded for data failings than from the Chris condition (61 excluded vs. 262 not excluded). This result suggests that the memory data presented below might be biased by this exclusion imbalance, a possible limitation that ought to be kept in mind when considering the implications of Study 4. However, working against this potential bias limitation are results from an additional regression analysis which showed that assignment to a referent group did not predict the number of intrusions $(p=.848)$. Moreover, this imbalance was equivalent across the 3 task conditions, so it cannot explain differences in recall patterns that might emerge in the $\mathrm{MN}$ replication condition and in the two conditions in which the behaviors were rated.

Free recall responses. Recall proportions were again calculated and entered into a mixed model ANOVA (means and standard deviations are listed in Table 6). We first considered whether we could safely combine the data from our two waves of data collection. We therefore conducted one 2 (Source: wave 1 vs. wave 2$) \times 2$ (Behavior referent: self vs. Chris) $\times 2$ (Trait type: central vs. peripheral) $\times 2$ (Behavior valence: positive vs. negative) mixed model ANOVA on the behavior recall data from the behavior rating condition, with source and behavior referent as between subject variable. We did the same analysis on the data from the behavior evaluation condition. The effect of the source of the data collection would be problematic if it affected the Behavior Referent $\times$ Tait Type $\times$ Behavior Valence interaction (behavior rating condition: $p=.955$; evaluation ratings condition: $p=.551$ ) or even the Behavior Referent $\times$ Behavior Valence interaction (behavior rating condition: $p=.702$; evaluation ratings condition: $p=.698$ ). This was not the case. Hence, we concluded that the data from the two waves of data collection could be safely combined. 
With this issue resolved, we turned to testing our main hypothesis. If making ratings would inhibit processing in a way that disrupts the mnemic neglect effect, we expected to find a 4-way interaction effect between condition, behavior referent, trait type and behavior valence. This was precisely what we found, $F(2,487)=3.77, p=.024, \eta_{p}{ }^{2}=.015$. Breaking down the interaction showed that the mnemic neglect-indicative three-way interaction between behavior referent, trait type, and behavior valence was not significant for the behavior rating condition $(p$ $=.134)$ or for the behavior evaluation condition $(p=.234)$ but only for the $\mathrm{MN}$ replication condition, $F(1,121)=3.94, p=.049, \eta_{p}{ }^{2}=.032$. Further breakdown in the MN replication condition showed the usual pattern for the mnemic neglect effect. The Behavior Valence $x$ Behavior Referent interaction was not significant for peripheral traits $(p=.317)$ and for central traits there was no significant difference in recall between self-referent and Chris-referent positive central trait-implying behaviors $(p=.307)$. In contrast, as is expected from the view that MN reflects a memory bias that follows from shallow processing, negative central trait-implying Chris-referent behaviors $(M=.25, S D=.17)$ were better recalled than negative central traitimplying behaviors that were self-referent $(M=.16, S D=.16), F(1,121)=10.20, p=.002, \eta_{p}{ }^{2}=$ .078 .

For completeness, we report (but do not discuss) those effects that were significant (or trending), but whose interpretations are qualified by the 4-way interaction: Trait Type $\times$ Behavior Valence $\times$ Condition $\left[F(2,487)=2.57, p=.078, \eta_{p}^{2}=.010\right]$, Trait Type $\times$ Behavior Valence $\left[F(1,487)=10.57, p=.001, \eta_{p}{ }^{2}=.021\right]$, Behavior Referent $\times$ Behavior Referent $\times$ Condition $\left[F(2,487)=2.98, p=.052, \eta_{p}{ }^{2}=.012\right]$, Behavior Valence $[F(1,487)=61.29, p<$ $\left..001, \eta_{p}{ }^{2}=.112\right]$, Trait Type $\left[F(1,487)=693.72, p<.001, \eta_{p}{ }^{2}=.588\right]$, Condition $[F(2,487)=$ $\left.2.70, p=.068, \eta_{p}{ }^{2}=.011\right]$, and Behavior Referent $\left[F(1,487)=6.51, p=.011, \eta_{p}{ }^{2}=.013\right]$. 
Behavior rating and behavior evaluation responses. The behavior rating task was introduced into the MN paradigm as a way of altering behavior processing. However, the ratings provided during this task may themselves be theoretically informative. There is an intriguing alternative explanation for MN that, to our knowledge, has never been explored. This explanation suggests that MN may be caused by differences in how important negative behaviors are perceived to be when they are performed by Chris vs. the self. For example, consider the behavior "kicked the dog." People may see that behavior as more negative when performed by Chris than when performed by the self. Stimulus extremity is known to be related to memory. Thus, it may be that central negative trait-relevant behaviors may be recalled more poorly for the self than for Chris behaviors simply because the perceived extremity of the behaviors differs for self and Chris. While behavior importance has been considered as a potential contributor to MN effects (Sedikides \& Green, 2000), Chris vs. self differences in other behavior characteristics (valence/extremity, informativeness, evaluation) do not seem to have been considered as potential contributors to MN. We did so in a series of additional analyses. Each of these measures was separately entered into a mixed model ANOVA reflecting the experimental design that we specified earlier. Descriptive results for the valence/extremity, informativeness, importance, and evaluation ratings all appear in Table 7. The ratings results were clear and consistent for the valence/extremity and importance rating variables (a complete walkthrough of the results appears in the online material that accompanies this article). The ratings never significantly shifted as a function of any analysis effect that contained the behavior referent variable (the effect that was closest was the interaction behavior referent and trait type for the valence rating, $p=.168$ ). Hence, these perceived behavior characteristics do not seem to be likely contributors to MN. Results with similar implications emerged for the evaluation ratings. 
The two crucial interactions that might reflect MN (Behavior Referent $\times$ Behavior Valence and Behavior Referent $\times$ Trait Type $\times$ Behavior Valence) were not significant $(p s>.13)$. Significant results for these ratings irrelevant to $\mathrm{MN}$, appear in the online material that accompanies the article.

However, the story for the informativeness ratings differs from the story told for the other 3 ratings. Interpretation of most significant effects produced by the informativeness analyses were qualified by the 3-way interaction described below. These effects will not be discussed, but are presented for completeness. These included effects for: Trait Type $[F(1,177)=207.01, p<$ $\left..001, \eta_{p}{ }^{2}=.539\right]$, Behavior Valence $\left[F(1,177)=9.20, p=.003, \eta_{p}{ }^{2}=.049\right]$, and Trait Type $\times$ Behavior Valence $\left[F(1,177)=23.12, p<.001, \eta_{p}{ }^{2}=.116\right]$.

For the informativeness ratings the interaction between trait type, behavior valence and behavior referent was significant, $F(1,177)=5.37, p=.022, \eta_{p}{ }^{2}=.029$. Breaking down the 3way interaction first by referent showed that there was a significant Trait Type $\times$ Behavior Valence interaction when the referent was Chris $\left[F(1,96)=4.00, p=.048, \eta_{p}{ }^{2}=.040\right]$ and also when the referent was the self $\left[F(1,81)=19.84, p<.001, \eta_{p}{ }^{2}=.197\right]$. Further breakdown showed that no significant results for behavior valence when peripheral traits were implied $(p s>$ $.15)$, but significant effects for behavior valence when central traits were implied, both for Chrisreferent behaviors (positive: $M=1.72, S D=0.77$; negative: $M=1.41, S D=1.29 ; F(1,96)=$ $5.81, p=.018, \eta_{p}{ }^{2}=.057$ ) and for self-referent behaviors (positive: $M=1.69, \mathrm{SD}=0.70$; negative: $\left.M=1.18, \mathrm{SD}=1.52 ; F(1,81)=10.92, p=.001, \eta_{p}{ }^{2}=.119\right)$.

The important result here shows that negative behaviors that were relevant to a central trait dimension were rated as less informative when they described the self than when they described Chris. This pattern maps on to the mnemic neglect effect, suggesting that differential 
perceived behavior informativeness for Chris and for the self might contribute to MN. Indeed, the result seems to support the notion that $\mathrm{MN}$ is linked to the extent to which people integrate or separate information from the self. The idea that the MN effect in memory might be linked to the perceived informativeness of a behavior was formally explored in a series of subsidiary analyses. In each analysis (executed on the data from each of the four studies), the perceived informativeness ratings obtained from Study 4 were entered into analyses as a predictor of performance on the memory task. This was accomplished in an initial regression analysis in which two variables (participants, designated as a categorical variable, and the informativeness ratings) predicted recall scores. This analysis allows examination of the informativenessmemory relation after extracting between-subjects variance (via the categorized participant variable). Next, for each study the residuals from this analysis were calculated and were used as the entries into a 2 (Behavior referent: self, Chris $) \times 2$ (Trait type: central, peripheral) $\times 2$ (Behavior valence: positive, negative) mixed model ANOVA. Each ANOVA was examined for evidence that the informativeness ratings could statistically account for MN effect in memory. Because we were looking for evidence that the informativeness ratings could statistically account for MN, we only performed the analysis on those main conditions of each study that essentially replicated the MN paradigm. This included Study 4's MN replication condition, Study 1's no delay condition, all the data from Study 2, and Study 3's no cognitive load condition.

For all four of these data sets, results from the initial regression analysis showed that after extracting between-subjects effects, higher informativeness ratings (obtained from Study 4) predicted better memory $($ Study $4: b=.243, t(489)=11.74, p<.001$; Study $1: b=.306, t(261)=$ 7.99, $p<.001 ;$ Study 2: $b=.363, t(353)=13.90, p<.001 ;$ Study $3:, b=.385, t(141)=9.14, p<$ .001. The residuals from the analysis of each data set from each regression were separately 
entered into a 2 (Behavior referent: self vs. Chris $) \times 2$ (Trait type: central vs. peripheral $) \times 2$ (Behavior valence: positive vs. negative) mixed model ANOVA. In 3 of these analyses (Study 4, Study 1 , Study 3 ), the Behavior Referent $\times$ Trait Type $\times$ Behavior Valence interaction that was significant in the original analysis of the memory data was not significant in the analysis of the residuals, (Study $4 p=.935$; Study $1 p=.630$; Study $3 p=.569$ ). These results are congruent with the idea that the perceived informativeness of the behaviors may play a role in behavior memory. However, the Behavior Referent $\times$ Trait Type $\times$ Behavior Valence interaction that was significant in the original analysis of the memory data from Study 2 remained significant in the analysis of the residuals, $F(1,87)=4.34, p=.04, \eta_{p}{ }^{2}=.084$. This last result suggests that the MN effect reflects processes that are not necessarily linked to the perceived informativeness of behaviors.

The lack of consistency in the results observed across the four studies might reflect the possibility that informativeness might merely be a correlate of $\mathrm{MN}$, not necessarily a cause of MN. In any case, our data are the first to highlight a link between the referent of important negative behavior and the perceived informativeness of behavior. Future MN research may want to pursue the implications of this link for the emergence of $\mathrm{MN}$.

\section{Discussion}

The results from Study 4 showed that asking people to deviate from the spontaneous manner in which they thought about the behaviors (by asking them to engage in a behavior rating task or behavior evaluation task) produced loss of MN. This outcome was expected from the standard MN processing model: Equalizing the extent to which all behaviors were processed by interfering with the extent to which people linked encountered behaviors to existing knowledge should eliminate $\mathrm{MN}$ in the free recall of self-referent/central trait-relevant/negative 
behaviors. It did. Moreover, because to provide ratings participants must read and understand all behaviors, the results from Study 4 argue against the possibility that loss of $\mathrm{MN}$ in the MN paradigm occurs because of inattention to, or incomplete understanding of, self-threatening behaviors. People have to read and understand the behaviors to rate them. Additionally, the sample sizes in both rating conditions were substantially larger than the sample size in the MN replication condition. Thus, it is unlikely that a loss of $\mathrm{MN}$ in these rating conditions is a consequence of a lack of power.

The collection of the behavior ratings also allowed assessment of the possibility that MN was related to changes in how the behaviors were perceived when they reflected the self versus when they reflected Chris. Examination of ratings of the extent to which events were negative or positive, were rated as important to personality to perform or not to perform, were evaluated as good or bad, did not support this change-in-perception view. However, this view was supported by the judgments of the informativeness of a behavior to personality: Central negative behaviors were seen as less informative to personality when the behaviors were self-descriptive than when they described Chris. Though not consistent across all four studies, results from subsidiary analyses suggested that referent-linked view of the informativeness of central negative behaviors might influence the MN effect. This could occur if the perceived characteristics of the behavior (informativeness for personality) affected how it is processed.

One limitation of the interpretation of the behavior ratings task is the way in which we phrased "important to personality to perform/not perform". As one of the reviewers of an earlier version of this article pointed out, the way we tried to rephrase the original items from Sedikides and Green (2000) to reflect a referent-independent description might have confused our participants. A review of comments left by participants at the end of the study confirmed that 
three participants indeed explicitly stated that they had found this particular question confusing. While this does not impact the effectiveness of the task on the recall assessment, it does limit the In addition, the procedure allows researchers to test more directly the differences between self-perception and other-perception, as the authors articulated usefulness of interpreting the responses to the question itself. However, the other two behavior ratings (and the evaluation rating) were not reported as confusing.

\section{Overall Discussion}

\section{Our Studies: Results Overview and Theoretical Implications}

The studies described in the present article pursued several goals. One goal of the present studies was to explore the scientific credibility of $\mathrm{MN}$ by repeatedly trying to replicate the phenomenon. A second goal was to explore the descriptive waxing and waning of $\mathrm{MN}$ : to expand on the corpus of knowledge that details when $\mathrm{MN}$ occurs, and when it does not. A third goal was to gain information about cognitive mechanisms that contribute to $\mathrm{MN}$ (or that do not contribute to $\mathrm{MN})$. Of particular interest was helping to more exactly specify what "shallow processing" of self-threating negative behaviors entailed: Ignoring (or inattention to) such behaviors or failure to integrate into existing self-knowledge. A fourth goal was to add to our knowledge about the general issue of bias in self-memory.

We accomplished three of these goals and made progress toward the fourth. When duplicating the procedure pioneered by Sedikides and Green (2000), we found evidence of bias in memory in the form of MN. Negative behaviors were recalled at a low rate when those behaviors were self-framed (as opposed to Chris-framed), especially when behaviors implied central traits (instead of peripheral traits). These results emerged: (a) when the free recall task immediately followed the behavior presentation; (b) even when there was a 48-hour delay 
between the behavior presentation and the free recall task; (c) regardless of whether participant processing of the behaviors was self-paced or computer-paced (assuming sufficient processing time); and (d) when the paradigm was implemented on the internet using samples of Mechanical Turk workers.

However, this MN bias did not always emerge. Loss of MN was observed when: (a) memory was assessed via a recognition task instead of via a free recall task; (b) people were asked to provide ratings or evaluations of the behaviors as they were encountered; (c) behavior encoding was accompanied by a classic cognitive load task.

The studies also provided correlational evidence about many variables that did not seem to contribute to $\mathrm{MN}$. MN was not linked to the time it took for people to read the behaviors. It was also not linked to perceptions of behavior valence/extremity, perceptions of the extent to which a behavior was important to perform, or the extent to which a behavior was perceived to be good or bad. However, MN was linked to the extent to which important negative behaviors are perceived to be informative about a person's personality.

The picture of MN that is emerging, then, is that the cascade of mental processes that lead to MN seems to originate during behavior encoding. Disrupting the way in which people usually think about the behaviors during behavior encoding causes loss of MN. However, this disruption does not seemingly involve ignoring or not attending to threatening behaviors, as might be suggested by a perceptual defense view (e.g., Erdelyi, 1974). What justifies this conclusion? It took about as long for people to read self-threatening negative behaviors as it did for them to read self-promoting positive behaviors. Moreover, $\mathrm{MN}$ was observed only in free recall, and not in recognition memory. Both findings suggest that people do attend to, and store in memory, important negative self-relevant information. 
Instead of inattention, the MN effect seems to be caused by the manner in which events are stored, which affects the ability to retrieve those events. What justifies this conclusion? MN occurs in free recall, but not in behavior recognition, implicating memory storage and search processes as a contributor to $\mathrm{MN}$. MN dissipates when a cognitive load is imposed during behavior processing. This effect occurs even when the load involves intensive processing of the behavior, as must occur when people provide various ratings of the behavior. All of these findings point to the idea that important self-negative memories are attended to, but they are not elaborated on in a manner that links such behaviors to existing self-knowledge. Hence, these negative memories are like "islands" in a sea of memories, with few bridges or ferries that can locate the memory during the kinds of memory search that occur during free recall.

\section{Looking to the Future of MN}

While the length of this list of contributions to the cognition of MN might seem impressive, much more remains to be done. For example, memory assessment is not limited to recognition and free recall: there are other methods of assessing memory. These include cued recall tasks, remember/know judgments, and implicit memory tasks. One way in which research into MN can be fruitfully expanded is to employ as many of these alternative memory assessments as possible. Doing so is desirable because it would help to verify that $\mathrm{MN}$ emerges primarily in those tasks that depend on memory search.

Additional contributions to an understanding of $\mathrm{MN}$ need to pursue additional details about the mental processes that lead to MN. For example, we remain intrigued by finding that instructions to "include behaviors in the self" produces loss of MN (Pinter et al., 2011), and wonder exactly how those instructions alter the usual processing that occurs in the $\mathrm{MN}$ paradigm. We also wonder whether $\mathrm{MN}$ reflects an inability to access important negative 
behaviors during a memory search, or whether $\mathrm{MN}$ reflects an unwillingness to do so. For example, what might happen if people were selectively rewarded for their performance in reporting the negative behaviors that they encountered? An increase in performance suggests that $\mathrm{MN}$ might, in part, reflect a lack of motivation to find the important self-relevant information that might be stored in memory.

The study of MN also needs to be expanded in other ways. Sedikides and Green (2000) did an exemplary job of pretesting stimuli for use in the MN paradigm. They did such a good job that subsequent studies of MN have almost exclusively used their stimuli (for an exception, see Zengel et al., 2015). This selectivity obviously poses a risk to the generality of MN. The theory of MN suggests that MN occurs in response to self-threatening stimuli. However, evidence for MN has emerged in studies using primarily socially-oriented traits and behaviors implying those traits. This social-trait focus ignores the fact that one fundamental idea in social psychology is that some behaviors imply traits related to competence. It is unknown whether failure behaviors will provide the same degree of self-threat as behaviors that impugn trustworthiness or kindness.

Indeed, there may be theoretical reasons to suspect that MN may differ for behaviors on the morality dimension and for behaviors on the competence dimension. For example, reasoning from Leary's sociometer idea (e.g., Leary, 2005), because traits such as unkindness or untrustworthy may be especially likely to lead to social rejection, any thoughts that one has about those traits might be especially threatening. In comparison, behaviors related to incompetence may be less likely to lead to rejection, and so might be relatively less self-threatening. On the other hand, some authors (see Wojciszke, 2005) have claimed that lack of competence poses an especially severe threat to the self. Thus, from the Wojciske view, it may be that MN is 
especially prevalent for behaviors that have self-incompetence implications. More broadly, this analysis suggests that there is much to be gained from expanding the range of stimuli used in the mnemic neglect paradigm so that they can be matched to the many domains that explore bias in self-memory.

\section{MN in its Broader Context: Considerations and Research Implications}

Moreover, we strongly encourage scholars to think about the phenomenon of mnemic neglect as it relates to the broader psychological context. For example, the $\mathrm{MN}$ effect can be linked to the general study of bias in self-memory (see Heinrichs \& Hofman, 2001; Zengel et al., 2015). In one example of such work, Skowronski (2011) considered two phenomena: a positivity bias in autobiographical memory and the fading affect bias (positive memories tend to prompt greater emotion at recall than do negative memories; see Skowronski, Walker, Henderson, \& Bond, 2014) in autobiographical memory. He speculated that both effects might reflect the action of common processes designed to keep individuals positive so that they can continue to engage in and explore the world around them. The mnemic neglect paradigm may fit with these phenomena, and because it is a laboratory paradigm, it is especially well-positioned to explore the positivity-promoting processes that cannot easily be explored in the nonexperimental autobiographical memory paradigms. Moreover, the results from much of the research in these real-world memory domains are confusing and contradictory, in part because the studies use real-world stimuli. The characteristics of those real-world stimuli are difficult to match across conditions. The beauty of the MN paradigm is that people in different conditions are processing the same behavioral stimuli, but in the context of different referents. This means that across referents, differences between stimuli cannot be the cause of the effects observed. The MN paradigm can ensure that observed effects are not caused by the many uninteresting 
methodological confounds that emerge from the use of existing real-world stimuli, but instead emanate from the manner in which perceivers process stimulus events.

Mnemic neglect research can also be linked two psychology's long-standing interest in differences in self-thought and other-thought. For example, considerable evidence suggests that people think differently about the self than they think about others (e.g., Beer \& Watson, 2009), and one manifestation of this tendency is the self-serving bias in attribution (for an example, see Krusemark, Campbell, \& Clementz, 2009). The mnemic neglect effect can be viewed as another example of these kinds of differences in self-thought and other-thought. Indeed, some real world autobiographical memory data suggests that people remember the best about themselves but the worst about others (see Thompson, et al., 1996). In this context, one puzzle in the MN data is why important self-framed positive information is not better recalled than the same information that describes the fictional other, Chris.

A final example of how mnemic neglect fits into the broad psychological context is the area of memory research exploring the self-reference effect (SRE): linking information to the self tends to enhance memory for the information (for example, see Bentley, Greenaway, \& Haslam, 2017). Another puzzle in the mnemic neglect paradigm is that it generally does not seem to evince this self-reference effect, though the fact that $\mathrm{MN}$ increases with separationrelated thought does fit with the mechanisms thought to drive the SRE.

These examples illustrate two ideas. First, the phenomenon of mnemic neglect is not simply a niche phenomenon. Instead, it can be linked to, and can potentially inform, research in a large number of related areas. The second idea illustrated by our contextual examples is that, when viewed in the context of other research areas, the data from the $\mathrm{MN}$ paradigm raises a large number of fascinating questions. Thus, there is a lot of research that needs to be done. We look 
forward to the new insights to be derived about self-processing, other-processing, and the nature of memory that can be gleaned from $\mathrm{MN}$ research. 


\section{References}

Banaji, M. R., \& Greenwald, A. G. (1995). Implicit gender stereotyping in judgments of fame. Journal of Personality and Social Psychology, 68, 181-198. doi:10.1037/00223514.68.2.181

Bargh, J. A., \& Thein, R. D. (1985). Individual construct accessibility, person memory, and the recall-judgment link: The case of information overload. Journal of Personality and Social Psychology, 49, 1129-1146. doi:10.1037/0022-3514.49.5.1129

Beer, A. \& Watson, D. (2008). Asymmetry in judgments of personality: Others are less differentiated than the self. Journal of Personality, 76, 535-559. doi: 10.1111/j.14676494.2008.00495.x

Bentley, S.V., Greenaway, K.H., \& Haslam, S. A. (2017). An online paradigm for exploring the self-reference effect. PLOS ONE, 12, ArtID e0176611. doi: 10.1371/journal.pone.0176611

Belmore, S. M. (1987). Determinants of attention during impression formation. Journal of Experimental Psychology: Learning, Memory, and Cognition, 13, 480-489. doi:10.1037/0278-7393.13.3.480

Brown, S. C., \& Craik, F. I. M. (2000). Encoding and retrieval of information. In E. Tulving \& F. I. M. Craik (Eds.), The Oxford handbook of memory (pp. 93-107). Oxford, UK: Oxford University Press.

Craik, F. I. M. (2002). Levels of processing: Past, present ... and future? Memory, 10, 305-318. doi:10.1080/09658210244000135

Erdelyi M. H. (1974). A New Look at the New Look: Perceptual Defense and Vigilance. Psychological Review, 81, 1-25. doi: 10.1037/h0035852 
Fiske, S.T. (1980). Attention and weight in person perception: The impact of negative and extreme behavior. Journal of Personality and Social Psychology, 38, 889-906. doi: $10.1037 / 0022-3514.38 .6 .889$

Green, J. D., Pinter, B., \& Sedikides, C. (2005). MN and self-threat: Trait modifiability moderates self-protection. European Journal of Social Psychology, 35, 225-235. doi:10.1002/ejsp.242

Green, J. D., \& Sedikides, C. (2004). Retrieval selectivity in the processing of self-referent information: Testing the boundaries of self-protection. Self and Identity, 3, 69-80. doi:10.1080/13576500342000059

Green, J. D., Sedikides, C., \& Gregg, A. P. (2008). Forgotten but not gone: The recall and recognition of self-threatening memories. Journal of Experimental Social Psychology, 44, 547-561. doi:10.1016/j.jesp.2007.10.006

Green, D. J., Sedikides, C., Pinter, B., \& Van Tongeren, D. R. (2009). Two sides to selfprotection: Self-improvement strivings and feedback from close relationships eliminate mnemic neglect. Self and Identity, 8, 233-250. doi: 10.1080/15298860802505145

Heinrichs, N., \& Hofman, S. G. (2001). Information processing in social phobia: A critical review. Clinical Psychology Review, 21, 751-770. doi:10.1016/S0272-7358(00)00067-2

Jones, L.L., \& Brunell, A.B. (2014). Clever and crude but not kind: Narcissism, self-esteem, and the self-reference effect. Memory, 22, 307-322. doi: 10.1080/09658211.2013.778999

Krusemark, E.A., Campbell, W. K., \& Clementz, B.A. (2008). Attributions, deception, and event related potentials: An investigation of the self-serving bias. Psychophysiology, 45, 511-515. doi: 10.1111/j.1469-8986.2008.00659.x 
Leary, M. R. (2005): Sociometer theory and the pursuit of relational value: Getting to the root of self-esteem. European Review of Social Psychology, 16, 75-111. doi: $10.1080 / 10463280540000007$

Loftus, G. (1985). Evaluating forgetting curves. Journal of Experimental Psychology: Learning, Memory, \& Cognition, 11, 397-406. doi: 10.1037/0278-7393.11.2.397.

Newman, L.S., Eccleston, C.P., \& Oikawa, M. (2017). Ignoring biased feedback: Membership in a stigmatized group as a moderator of mnemic neglect. The Journal of Social Psychology, 157, 152-164. doi: 10.1080/002245.2016.1176550

Newman, L. S., Nibert, J. A., \& Winer, E. S. (2009). MN is not an artifact of expectancy: The moderating role of defensive pessimism. European Journal of Social Psychology, 39, 477-486. doi:10.1002/ejsp.598

Newman, L. S., Sapolsky, M. S., Tang, Y., \& Bakina, D. A. (2014). What's recalled depends on the nature of the recall procedure. Social Psychology, 45, 93-102. doi: 10.1027/18649335/a000164

Klein, S. B. (2012). Self, memory, and the self-reference effect: An examination of conceptual and methodological issues. Personality and Social Psychology Review, 16, 283-300. doi:10.11771088868311434214

Ochsner, K.N. and Gross, J.J. (2005). The cognitive control of emotion. Trends in Cognitive Science, 9, 242-249. doi:10.1016/j.tics.2005.03.010

Pinter, B. Green, J.D., Sedikides, C., \& Gregg, A. P. (2011). Self-protective memory: Separation/integration as a mechanism for MN. Social Cognition, 29, 612-624. doi:10.1521/soco.2011.29.5.612 
Pool, E., Brosch, T., Delplanque, S., \& Sander, D. (2016). Attentional bias for positive emotional stimuli: A meta-analytic investigation. Psychological Bulletin, 142, 79-106. doi: $10.1037 / \mathrm{bul} 0000026$.

Roozendaal B. (2002). Stress and memory: opposing effects of glucocorticoids on memory consolidation and memory retrieval. Neurobiology of Learning \& Memory, 78, 578595. doi:10.1006/nlme.2002.4080

Saunders, J. (2011). Reversed memory neglect of self-threatening memories in dysphoria. Cognition and Emotion, 25, 854-867. doi: 10.1080/02699931.2010.524037

Saunders, J. (2012). Selective memory bias for self-threatening memories in trait anxiety. Cognition and Emotion, 27, 21-36. doi: 10.1080/02699931.2012.683851

Saunders, J., Vallath, S., \& Reed, P. (2015). Disruptions to processing of self-referential emotional material are associated with positive symptoms of schizotypy. Psychiatry Research, 229, 809-813. doi: 10.1016/j.psychres.2015.07.086

Saunders, J., Worth, R., \& Fernandes, M. (2012). Repressive coping style and MN. Journal of Experimental Psychopathology, 3, 346-367. doi: 10.5127/jep.020211

Sedikides, C., \& Green, J. D. (2000). On the self-protective nature of inconsistency - negativity management: Using the person memory paradigm to examine self-referent memory. Journal of Personality and Social Psychology, 79, 906-922. doi:10.1037//00223514.79.6.906

Sedikides, C., Green, J. D., \& Pinter, B. (2004). Self-protective memory. In D. R., Beike, J. M. Lampinen, \& D. A. Behrend (Eds.), The self and memory (pp. 161-179). New York, PA: Psychology Press. 
Sedikides, C., Green, J.D., Saunders, J., Skowronski, J.J., \& Zengel, B. (2016). Mnemic neglect: Selective amnesia of one's faults. European Review of Social Psychology, 27, 1-62, doi: $10.1080 / 10463283.2016 .1183913$

Sedikides, C., \& Strube, M. J. (1997). Self evaluation: To thine own self be good, to thine own self be sure, to thine own self be true, and to thine own self be better. In M.P. Zanna (Ed.), Advances in experimental social psychology (Vol. 29, pp. 209-269). San Diego, CA: Academic Press.

Schooler, J. W. (2014). Metascience could rescue the 'replication crisis'. Nature, 515, 9. doi:10.1038/515009a

Schnurr, P.P. \& Jankowski, M.K. (1999). Physical health and post-traumatic stress disorder: Review and synthesis. Seminars in Clinical Neuropsychiatry, 4, 295-304. doi: 10.153/SCNP00400295

Skowronski, J.J. (2011). The positivity bias and the fading affect bias in autobiographical memory: A self-motives perspective. In C. Sedikides \& M. Alicke (Eds.), The Handbook of self-enhancement and self-protection (pp. 211-231). New York: Guilford Press.

Skowronski, J. J., Walker, W. R., Henderson, D. X., \& Bond, G. D. (2014). The fading affect bias: Its history, its implications, and its future. In J. M. Olson and M. P. Zanna (Eds). Advances in Experimental Social Psychology (Vol. 49, pp. 163-218). Burlington: Academic Press.

Stanislaw, H. \& Todorov, N. (1999). Calculation of signal detection theory measures. Behavior Research Methods, Instruments and Computers, 31, 137-149. doi:10.3758/BF03207704 
Stern, L. D., Marrs, S., Millar, M. G., \& Cole, E. (1984). Processing time and the recall of inconsistent and consistent behaviors of individuals and groups. Journal of Personality and Social Psychology, 47, 253-262. doi:10.1037/0022-3514.47.2.253

Swets, J. A. (1996). Signal detection theory and ROC analysis in psychology and diagnostics: Collected papers. Mahwah, NJ: Erlbaum.

Thompson, C. P., Skowronski, J. J., Larsen, S. F., \& Betz, A. L. (1996). Autobiographical memory: Remembering what and remembering when. Mahwah, NJ: Erlbaum

Wells, B. M., Skowronski, J. J., Crawford, M. T., Scherer, C. R., \& Carlston, D. E. (2011). Inference making and linking both require thinking: Spontaneous trait inference and spontaneous trait transference both rely on working memory capacity. Journal of Experimental Social Psychology, 47, 1116-1126. doi:10.1016/j.jesp.2011.05.013

Wojciszke, B. (2005). Morality and competence in person- and self-perception. European Review of Social Psychology, 16, 155-188. doi: 10.1080/10463280500229619

Wyer, R. S. Jr., \& Carlston, D.E. (1979). Social cognition, inference, and attribution. Hillsdale, NJ: Lawrence Erlbaum Publishers.

Zengel, B., Skowronski, J. J., Valentiner, D. P., \& Sedikides, C. (2015). Loss of MN among socially anxious individuals. Journal of Social and Clinical Psychology, 34, 322-347. doi: $10.1521 /$ jscp.2015.34.4.322 


\section{Footnotes}

${ }^{1}$ To be clear, two of the manuscript's authors (BZ and JJS) have previously collaborated with Dr. Sedikides. In addition, as we pursued the studies described in the present article, Dr. Sedikides generously provided stimuli and unpublished prestest results to us, and Dr. Green responded to many queries about the paradigm and about past results. However, the work that we report in this article is entirely our own; neither Dr. Sedikides or Dr. Green directly contributed to the design, conduct, analysis, or writeup of any of the studies reported in the present article.

${ }^{2}$ Amazon's Mechanical Turk (http://www.MTurk.com) is an online labor market that is designed to pay people small amounts of money in exchange for completing tasks that are simple for humans but difficult for computers. Examples of these Human Intelligence Tasks (HITs) range from choosing the best among several photographs of a storefront to writing product descriptions. More recently, however, MTurk has become popular within psychology and other social sciences as a means for online data collection (Buhrmester, Kwang, \& Gosling, 2011). MTurk is an especially attractive alternative to classical laboratory research, as it boasts a large, diverse workforce consisting of over 100,000 users from over 100 countries who complete tens of thousands of HITs daily (Pontin, 2007).

${ }^{3} G^{*}$ Power software (Version 3.1.2) was used to compute the required sample size given $\alpha(.05)$, power (.95), and variable effect sizes. Given the extant mnemic neglect literature, coupled with results from the Pilot Study, effect sizes for Referent $\times$ Trait Type $\times$ Behavior Valence interactions were expected to be large (effect size $\mathrm{f}=.5$ ). Assuming small to moderate effect sizes for the 4-way interactions proposed in this dissertation (e.g., Cognitive Load $\times$ Referent $\times$ Trait Type $\times$ Behavior Valence), hypothetical required sample sizes are shown in Table 11 . 
Required Sample Sizes for Proposed Dissertation Experiments

\begin{tabular}{cc}
\hline Effect Size $\mathrm{f}$ & Total Sample Size \\
\hline .20 & 132 \\
.25 & 86 \\
.30 & 60 \\
\hline
\end{tabular}

Based upon this power analysis, a sample size of at least 60 valid participants was deemed sufficient to adequately examine each of the hypothesized 4-way interactions. Data was deemed invalid if no behaviors were recalled or three or more intrusions were included. We aimed to oversample to ensure that the number of valid participants was sufficient.

${ }^{4}$ As is typical (e.g., Ratcliff, 1993), reading times were positively skewed and leptokurtotic ( $M=$ 4421; $S D=2876$; skewness $=4.11 ;$ kurtosis $=39.38)$. We applied many of the standard data analysis techniques that are often used in response to data that exhibits these characteristics. For example, conclusions drawn from analyses that entered log transformed reading times into the analysis to minimize these problems did not differ from the conclusions of the analyses reported in the text. Similar results also came from other data analyses, including analyses that removed outliers from the analysis and an analysis that used a dependent measure that controlled for sentence length by dividing the reading time for each sentence by the number of syllables in each sentence. Thus, for clarity and simplicity, all analyses described herein use raw reading time data.

${ }^{5}$ Our interpretation of the Study 3 data is also supported by results of analyses from data only in the no load condition. These analyses yielded a Behavior Referent $\times$ Trait Type $\times$ Behavior Valence interaction, $F(1,34)=4.04, p=.052, \eta_{p}{ }^{2}=.106$. Decomposing this interaction showed that the Behavior Referent $\times$ Behavior Valence interaction did not emerge for peripheral traitimplicative behaviors $(p=.525)$. However, this interaction did emerge for central trait- 
implicative behaviors, $F(1,34)=4.79, p=.036, \eta_{p}{ }^{2}=.123$. Further decomposition showed that there was no significant difference in recall for self-referent compared to Chris-referent positive central trait-implicative behaviors $(p=.244)$. However, self-referent negative central traitimplicative behaviors were recalled less well than Chris-referent negative central traitimplicative behaviors, $F(1,34)=3.11, p=.087, \eta_{p}{ }^{2}=.084$. 
Table 1

Proportion of Behaviors Recalled $(S D)$ for the Recall Delay $\times$ Behavior Referent $\times$ Trait Type $\times$ Behavior Valence Interaction (Excluding Intrusions; $N=129$ ) in Study 1

\begin{tabular}{lcccc}
\hline & \multicolumn{2}{c}{ Central Behaviors } & \multicolumn{2}{c}{ Peripheral Behaviors } \\
Behavior Referent & Positive & Negative & Positive & Negative \\
\hline No Recall Delay & $.36(.20)$ & $.25(.23)$ & $.16(.21)$ & $.13(.19)$ \\
$\quad$ Self & $.38(.16)$ & $.39(.24)$ & $.17(.14)$ & $.17(.13)$ \\
$\quad$ Chris & & & & \\
48-Hour Recall Delay & $.22(.16)$ & $.08(.15)$ & $.05(.12)$ & $.06(.08)$ \\
$\quad$ Self & $.18(.14)$ & $.20(.11)$ & $.07(.09)$ & $.10(.16)$ \\
$\quad$ Chris &
\end{tabular}


Table 2

Behavior Recognition Accuracy $(S D)$ for the Recall Delay $\times$ Behavior Referent $\times$ Trait Type $\times$ Behavior Valence Interaction (Excluding Intrusions; $N=129$ ) in Study 1

\begin{tabular}{lcccc}
\hline & \multicolumn{2}{c}{ Central Behaviors } & \multicolumn{2}{c}{ Peripheral Behaviors } \\
Behavior Referent & Positive & Negative & Positive & Negative \\
\hline No Recall Delay & & & & \\
$\quad$ Self & $.79(.17)$ & $.79(.14)$ & $.76(.14)$ & $.78(.15)$ \\
Chris & $.85(.16)$ & $.85(.15)$ & $.77(.16)$ & $.82(.14)$ \\
48-Hour Recall Delay & & & & \\
$\quad$ Self & $.69(.16)$ & $.71(.15)$ & $.67(.15)$ & $.70(.15)$ \\
$\quad$ Chris & $.78(.11)$ & $.76(.12)$ & $.73(.10)$ & $.71(.12)$ \\
\hline
\end{tabular}

Note. Behavior recognition accuracy values $(\delta)$ were derived by converting mean hits and mean correct rejections into proportions, and then by averaging the result, for each set of eight behaviors defined by the interaction of Trait Type and Behavior Valence. 
Table 3

Proportion of Behaviors Recalled (SD) for the Behavior Referent $\times$ Trait Type $\times$ Behavior Valence Interaction (Excluding Intrusions; $N=89$ ) in Study 2

\begin{tabular}{lllll}
\hline & \multicolumn{2}{c}{ Central Behaviors } & & \multicolumn{2}{c}{ Peripheral Behaviors } \\
& Positive & Negative & Positive & Negative \\
\hline Behavior Referent & & & & \\
$\quad$ Self & $.41(.15)$ & $.21(.14)$ & $.11(.11)$ & $.13(.13)$ \\
Chris & $.40(.17)$ & $.39(.18)$ & $.20(.19)$ & $.17(.14)$ \\
\hline
\end{tabular}


Table 4

Behavior Reading Time Means $(S D)$ for the Behavior Referent $\times$ Trait Type $\times$ Behavior Valence Interaction (Excluding Intrusions; $N=89$ ) in Study 2

\begin{tabular}{ccccc}
\hline & \multicolumn{2}{c}{ Central Behaviors } & & \multicolumn{2}{c}{ Peripheral Behaviors } \\
& Positive & Negative & Positive & Negative \\
\hline Behavior Referent & & & \\
Self & 4605 (1722) & $4081(1383)$ & $4612(1747)$ & $4517(1600)$ \\
Chris & 4681 (1466) & 3978 (1276) & 4476 (1373) & $4410(1356)$ \\
\hline \multicolumn{2}{l}{ Note. Means and standard deviations are reported in milliseconds. }
\end{tabular}


Table 5

Proportion of Behaviors Recalled (SD) for the Cognitive Load $\times$ Behavior Referent $\times$ Trait Type $\times$ Behavior Valence Interaction (Excluding Intrusions; $N=67$ ) in Study 3

\begin{tabular}{|c|c|c|c|c|}
\hline \multirow[b]{2}{*}{ Behavior Referent } & \multicolumn{2}{|c|}{ Central Behaviors } & \multicolumn{2}{|c|}{$\underline{\text { Peripheral Behaviors }}$} \\
\hline & Positive & Negative & Positive & Negative \\
\hline \multicolumn{5}{|l|}{ No Cognitive Load } \\
\hline Self & $.45(.15)$ & $.29(.18)$ & $.13(.14)$ & $.12(.13)$ \\
\hline Chris & $.39(.15)$ & $.40(.19)$ & $.20(.14)$ & $.16(.14)$ \\
\hline \multicolumn{5}{|l|}{ Cognitive Load } \\
\hline Self & $.15(.11)$ & $.26(.19)$ & $.09(.11)$ & $.06(.08)$ \\
\hline Chris & $.23(.19)$ & $.18(.12)$ & $.06(.10)$ & $.09(.08)$ \\
\hline
\end{tabular}


Table 6

Proportion of Behaviors Recalled (SD) for the Behavior Referent $\times$ Trait Type $\times$ Behavior Valence Interaction in Study 4

\begin{tabular}{lcccc}
\hline & \multicolumn{2}{c}{ Central Behaviors } & \multicolumn{2}{c}{ Peripheral Behaviors } \\
Behavior Referent & Positive & Negative & Positive & Negative \\
\hline MN Replication & & & & \\
$\quad$ Self & $.24(.17)$ & $.16(.16)$ & $.08(.12)$ & $.05(.08)$ \\
$\quad$ Chris & $.27(.16)$ & $.25(.17)$ & $.13(.15)$ & $.08(.10)$ \\
\hline Behavior Rating & & & & \\
$\quad$ Self & $.30(.21)$ & $.23(.19)$ & $.11(.13)$ & $.08(.12)$ \\
$\quad$ Chris & $.31(.19)$ & $.23(.15)$ & $.11(.14)$ & $.13(.14)$ \\
\hline Evaluation Ratings & & & & \\
$\quad$ Self & $.28(.17)$ & $.25(.17)$ & $.11(.15)$ & $.09(.12)$ \\
$\quad$ Chris & $.33(.17)$ & $.24(.18)$ & $.12(.14)$ & $.08(.10)$ \\
\hline
\end{tabular}


Table 7

Valence Ratings (SD), Importance Ratings (SD), Informativeness Ratings (SD), and Evaluation Ratings $(S D)$ for the Behavior Referent $\times$ Trait Type $\times$ Behavior Valence Interaction in Study 4

\begin{tabular}{lcccc}
\hline & \multicolumn{2}{c}{ Central Behaviors } & & \multicolumn{2}{c}{ Peripheral Behaviors } \\
Behavior Referent & Positive & Negative & Positive & Negative \\
\hline Valence/Extremity & & & & \\
Rating & & & & \\
$\quad$ Self & $2.03(0.60)$ & $-2.28(0.60)$ & $0.66(0.66)$ & $-1.44(0.56)$ \\
$\quad$ Chris & $2.08(0.66)$ & $-2.23(0.49)$ & $0.84(0.74)$ & $-1.37(0.53)$ \\
& & & & \\
Importance Rating & & & & \\
$\quad$ Self & $1.65(0.67)$ & $-1.87(0.90)$ & $0.50(0.65)$ & $-1.21(0.68)$ \\
$\quad$ Chris & $1.71(0.70)$ & $-1.59(1.11)$ & $0.65(0.61)$ & $-1.00(0.86)$ \\
$\quad$ & & & \\
Informativeness & & & & \\
Rating & & & & \\
$\quad$ Self & $1.69(0.70)$ & $1.18(1.52)$ & $0.90(0.77)$ & $0.97(1.08)$ \\
$\quad$ Chris & $1.72(0.77)$ & $1.41(1.29)$ & $1.07(0.69)$ & $0.96(0.99)$ \\
$\quad$ & & & \\
$\quad$ & & & & \\
$\quad$ Evaluation Rating & & & & \\
$\quad$ Self & $2.12(0.62)$ & $-2.13(0.74)$ & $0.74(0.63)$ & $-1.20(0.68)$ \\
$\quad$ Chris & $2.26(0.54)$ & $-2.22(0.50)$ & $0.92(0.62)$ & $-1.22(0.56)$ \\
\hline
\end{tabular}


Computer Screen 1

Computer Screen 2

Computer Screen 3

\begin{tabular}{|c|c|c|c|c|}
\hline $\begin{array}{l}\text { Remember the } \\
\text { following number: } \\
187359\end{array}$ & $\begin{array}{c}5 \text { seconds } \\
\text { pass }\end{array}$ & $\begin{array}{l}\text { "X would borrow } \\
\text { other people's } \\
\text { belongings without }\end{array}$ & $\begin{array}{c}8 \text { seconds } \\
\text { pass }\end{array}$ & $\begin{array}{l}\text { Please type in the } \\
\text { number you were } \\
\text { asked to remember: }\end{array}$ \\
\hline $18 / 359$ & & Knowledge. & & \\
\hline
\end{tabular}

Figure 1. Depiction of cognitive load manipulation in Study 3. 


\section{ONLINE MATERIAL}

\section{Study 4: Significant results of ratings analyses}

\section{Valence/Extremity Rating:}

Trait Type: $F(1,177)=67.02, p<.001, \eta_{p}{ }^{2}=.275$

Central: $M=-0.10, S D=0.29$

Peripheral: $M=-0.32, S D=0.38$

Behavior Valence: $F(1,177)=2257.61, p<.001, \eta_{p}{ }^{2}=.927$

Positive: $M=1.41, S D=0.59$

Negative: $M=-1.83, S D=0.46$

Behavior Referent: $F(1,177)=4.85, p=.029, \eta_{p}{ }^{2}=.027$

Self: $M=-0.26, S D=0.24$

Chris: $M=-0.17, S D=0.30$

Trait Type $\times$ Behavior Valence: $F(1,177)=946.15, p<.001, \eta_{p}{ }^{2}=.842$

Central - Behavior Valence: $F(1,178)=3185.14, p<.001, \eta_{p}{ }^{2}=.947$

Positive: $M=2.06, S D=0.63$

Negative: $M=-2.25, S D=0.54$

Peripheral - Behavior Valence: $F(1,178)=812.19, p<.001, \eta_{p}{ }^{2}=.820$

Positive: $M=0.76, S D=0.71$

Negative: $M=-1.40, S D=0.54$ 


\section{Importance Rating:}

Trait Type: $F(1,177)=63.75, p<.001, \eta_{p}{ }^{2}=.265$

Central: $M=-0.02, S D=0.52$

Peripheral: $M=-0.26, S D=0.47$

Behavior Valence: $F(1,177)=930.55, p<.001, \eta_{p}{ }^{2}=.840$

Positive: $M=1.13, S D=0.56$

Negative: $M=-1.41, S D=0.84$

Behavior Referent: $F(1,177)=6.74, p=.010, \eta_{p}{ }^{2}=.037$

Self: $M=-0.23, S D=0.35$

Chris: $M=-0.06, S D=0.51$

Trait Type $\times$ Behavior Valence: $F(1,177)=436.59, p<.001, \eta_{p}{ }^{2}=.712$

Central - Behavior Valence: $F(1,178)=1058.61, p<.001, \eta_{p}{ }^{2}=.856$

Positive: $M=1.69, S D=0.68$

Negative: $M=-1.72, S D=1.02$

Peripheral - Behavior Valence: $F(1,178)=447.33, p<.001, \eta_{p}{ }^{2}=.715$

Positive: $M=0.58, S D=0.63$

Negative: $M=-1.09, S D=0.79$

\section{Informativeness Rating:}

Trait Type: $F(1,177)=207.01, p<.001, \eta_{p}{ }^{2}=.539$

Central: $M=1.51, S D=0.89$

Peripheral: $M=0.98, S D=0.80$ 
Behavior Valence: $F(1,177)=9.20, p=.003, \eta_{p}^{2}=.049$

Positive: $M=1.35, S D=0.65$

Negative: $M=1.13, S D=1.17$

Trait Type $\times$ Behavior Valence: $F(1,177)=23.12, p<.001, \eta_{p}{ }^{2}=.116$

Central - Behavior Valence: $F(1,178)=16.31, p<.001, \eta_{p}{ }^{2}=.084$

Central - Positive: $M=1.71, S D=0.74$

Central - Negative: $M=1.30, S D=1.40$

Peripheral - Behavior Valence: $p=.598$

Trait Type $\times$ Behavior Valence $\times$ Behavior Referent: $F(1,177)=5.37, p=.022$,

$\eta_{p}^{2}=.029$

Chris - Trait Type $\times$ Behavior Valence: $F(1,96)=4.00, p=.048, \eta_{p}{ }^{2}=.040$

Chris \& Central - Behavior Valence: $F(1,96)=5.81, p=.018, \eta_{p}{ }^{2}=.057$

Positive: $M=1.72, S D=0.77$

Negative: $M=1.41, S D=1.29$

Chris \& Peripheral - Behavior Valence: $p=.150$

Self - Trait Type $\times$ Behavior Valence: $F(1,81)=19.84, p<.001, \eta_{p}{ }^{2}=.197$

Self \& Central - Behavior Valence: $F(1,81)=10.92, p=.001, \eta_{p}{ }^{2}=.119$

Positive: $M=1.69, \mathrm{SD}=0.70$

Negative: $M=1.18, \mathrm{SD}=1.52$

Self \& Peripheral - Behavior Valence: $p=.439$ 


\section{Evaluation Ratings:}

Trait Type: $F(1,189)=55.61, p<.001, \eta_{p}{ }^{2}=.227$

Central: $M=0.01, S D=0.24$

Peripheral: $M=-0.19, S D=0.37$

Trait Valence: $F(1,189)=2115.39, p<.001, \eta_{p}{ }^{2}=.918$

Positive: $M=1.52, S D=0.51$

Negative: $M=-1.69, S D=0.58$

Trait Type $\times$ Trait Valence: $F(1,189)=1353.52, p<.001, \eta_{p}{ }^{2}=.877$

Central - Behavior Valence: $F(1,190)=2994.02, p<.001, \eta_{p}{ }^{2}=.940$

Positive: $M=2.19, S D=0.58$

Negative: $M=-2.18, S D=0.62$

Peripheral - Behavior Valence: $F(1,190)=789.09, p<.001, \eta_{p}{ }^{2}=.806$

Positive: $M=0.84, S D=0.63$

Negative: $M=-1.21, S D=0.62$ 